\title{
Agentes inibidores da fosfodiesterase no tratamento da sepse e choque séptico
}

\author{
Phosphodiesterase inhibitors in the treatment of sepsis and septic shock \\ Inhibidores de la fosfodiesterasa en el tratamiento de la sepsis y el shock séptico
}

Recebido: 22/01/2021 | Revisado: 24/01/2021 | Aceito: 25/01/2021 | Publicado: 31/01/2021

\author{
Gabrielle Delfrate \\ ORCID: https://orcid.org/0000-0002-4326-9566 \\ Universidade Estadual de Ponta Grossa, Brasil \\ E-mail: g_delfrate@hotmail.com \\ Daniel Fernandes \\ ORCID: https://orcid.org/0000-0002-8935-4176 \\ Universidade Federal de Santa Catarina, Brasil \\ E-mail: fernandes.d@ufsc.br \\ Gilson Cesar Nobre Franco \\ ORCID: https://orcid.org/0000-0001-7082-7837 \\ Universidade Estadual de Ponta Grossa, Brasil \\ E-mail: gilsoncnf@gmail.com
}

\begin{abstract}
Resumo
A sepse é uma síndrome prevalente definida como uma disfunção orgânica com risco de vida causada por uma resposta desregulada do hospedeiro a uma infecção, podendo manifestar-se de diversas formas. A fisiopatologia da síndrome envolve as vias inflamatória e imunológica, sendo que a resposta do sistema circulatório exerce um papel fundamental na isquemia e lesão orgânica. Os fármacos inibidores da fosfodiesterase (iPDE) agem aumentando os níveis dos nucleotídeos cíclicos (AMPc e/ou GMPc). Esses agentes podem causar vários efeitos de acordo com a sua seletividade, entre eles: vasodilatação, aumento do débito cardíaco, diminuição da permeabilidade endotelial, inibição de citocinas pró-inflamatórias, e inibição da coagulação. Diante disso, foi levantado a hipótese de que os iPDE poderiam ser benéficos no tratamento da sepse. Estudos clínicos que utilizaram agentes iPDE no tratamento da sepse demonstram resultados promissores. Modelos animais têm sido utilizados para a investigação dos mecanismos envolvidos na patologia da sepse, bem como novas opções terapêuticas. A enzima fosfodiesterase é classificada em 11 famílias, sendo que as pesquisas são focadas nos fármacos inibidores não seletivos da PDE, iPDE-3, iPDE-4 e iPDE-5. Dois modelos sépticos principais são utilizados: aplicação de lipopolissácarídeo de Escherichia coli e cirurgia de ligadura e punção cecal. Os medicamentos são administrados de forma intraperitoneal ou intravenosa antes os depois da indução de sepse, em diferentes doses. Apesar do grande número de resultados positivos, as diferenças encontradas entre os modelos sépticos evidenciam a necessidade de padronização de métodos para a obtenção de dados confiáveis que possam ser utilizados na pesquisa clínica.
\end{abstract}

Palavras-chave: Terapia medicamentosa; Inibidores da fosfodiesterase; Inflamação; Experimentação animal.

\begin{abstract}
Sepsis is a prevalent syndrome defined as a life-threatening organ dysfunction caused by a host's unregulated response to an infection, which can manifest itself in several ways. The pathophysiology of the syndrome involves the inflammatory and immunological pathways, and the response of the circulatory system plays a fundamental role in ischemia and organic injury. Phosphodiesterase inhibitors (iPDE) act by increasing the levels of cyclic nucleotides (cAMP and / or cGMP). These agents promote several effects according to their selectivity, among them: vasodilation, increased cardiac output, decreased endothelial permeability, inhibition of pro-inflammatory cytokines, and inhibition of coagulation. In view of this, the hypothesis was raised that iPDE could be beneficial in the treatment of sepsis. Clinical studies that used iPDE agents to treat sepsis show promising results. Animal models have been used to investigate the mechanisms involved in the pathology of sepsis, as well as new therapeutic options. The phosphodiesterase enzyme is classified in 11 families, and the research is focused on non-selective PDE inhibitor drugs, iPDE-3, iPDE-4 and iPDE-5. Two main septic models are used: application of Escherichia coli lipopolysaccharide and ligation and cecal puncture surgery. The drugs are administered intraperitoneally or intravenously before sepsis induction, in different doses. Despite the large number of positive results, the differences found between the septic models show the need for standardization of methods to obtain reliable data that can be used in clinical research.
\end{abstract}

Keywords: Drug therapy; Phosphodiesterase inhibitors; Inflammation; Animal experimentation.

\section{Resumen}

La sepsis es un síndrome prevalente definido como una disfunción orgánica potencialmente mortal causada por la respuesta no regulada de un huésped a una infección, que puede manifestarse de varias formas. La fisiopatología del 
síndrome involucra las vías inflamatoria e inmunológica, y la respuesta del sistema circulatorio juega un papel fundamental en la isquemia y lesión orgánica. Los fármacos inhibidores de la fosfodiesterasa (iPDE) actúan aumentando los niveles de nucleótidos cíclicos (cAMP y / o cGMP). Estos agentes pueden producir diversos efectos según su selectividad, entre ellos: vasodilatación, aumento del gasto cardíaco, disminución de la permeabilidad endotelial, inhibición de citocinas proinflamatorias e inhibición de la coagulación. En vista de esto, se planteó la hipótesis de que el iPDE podría ser beneficioso en el tratamiento de la sepsis. Los estudios clínicos que utilizaron agentes iPDE para tratar la sepsis muestran resultados prometedores. Se han utilizado modelos animales para investigar los mecanismos implicados en la patología de la sepsis, así como nuevas opciones terapéuticas. La enzima fosfodiesterasa se clasifica en 11 familias y la investigación se centra en fármacos inhibidores de la PDE no selectivos, iPDE-3, iPDE-4 e iPDE-5. Se utilizan dos modelos sépticos principales: aplicación de lipopolisacárido de Escherichia coli y cirugía de ligadura y punción cecal. Los fármacos se administran por vía intraperitoneal o intravenosa antes de la inducción de la sepsis, en diferentes dosis. A pesar de la gran cantidad de resultados positivos, las diferencias encontradas entre los modelos sépticos muestran la necesidad de estandarizar los métodos para obtener datos confiables que puedan ser utilizados en la investigación clínica.

Palabras clave: Farmacoterapia; Inibidores de la fosfodiesterasa; Inflamación; Experimentación animal.

\section{Introdução}

A sepse é caracterizada como uma resposta inflamatória sistêmica a um foco infeccioso que afeta principalmente pacientes internados em situação de imunossupressão, como por exemplo pacientes em tratamentos oncológicos e idosos com comorbidades (Gotts \& Matthay, 2016). A síndrome é definida como uma disfunção orgânica com risco de vida causada por uma resposta desregulada do hospedeiro a uma infecção (Singer et al., 2016). O quadro séptico é complexo e envolve várias vias patogênicas. O conjunto de alterações circulatórias, celulares e metabólicas podem levar ao quadro de choque séptico, com risco de mortalidade maior. Apesar de muitos tratamentos terem sido estudados ao longo dos anos, ainda não há consenso sobre o manejo adequado dessas síndromes, sendo que as intervenções são baseadas na remoção da fonte de infecção, terapia antimicrobiana, restauração da perfusão do tecido e resolução da disfunção orgânica (Cecconi et al., 2018).

O monofosfato cíclico de adenosina (AMPc) e o monofosfato cíclico de guanosina (GMPc) são nucleotídeos envolvidos em várias vias fisiológicas. O aumento dos níveis de AMPc/GMPc está relacionado a vasodilatação, diminuição de resitência vascular periférica e diminuição de citocinas pró-inflamatórias (Németh et al., 1997; Fakioglu et al., 2004). Esses nucleotídeos são metabolizados pelas enzimas fosfodiesterases (PDE), a qual é dividida em 11 famílias com diferentes especificidades de substrato. Diante disso, a inibição da PDE pode ser uma estratégia para prevenir lesão isquêmica e diminuir a resposta inflamatória em pacientes sépticos (Kovalski et al., 2017; Pan et al., 2019).

Há uma variedade de medicamentos que agem como inibidores de PDE (iPDE) no mercado. Os iPDE-3, como amrinona, milrinona e oprinona e inibidores inespecíficos, como a pentoxifilina e teofilina, foram pioneiros nas pesquisas préclínicas e clínicas do manejo da sepse (Németh et al., 1997; Schimidt et al., 2000a; Miyakawa et al., 2004). Nos últimos anos, foram realizados estudos em modelos animais com o inibidor da PDE-5 sildenafil (Kovalski et al., 2017; Özer, Topal \& Sem, 2020) e iPDE-4 como rolipram e roflumilaste (Sims et al., 2017; Lu et al., 2020; Xu et al., 2020). Devido as recentes descobertas, e visto que a sepse e o choque representam um problema de saúde de alta incidência e difícil manejo, o objetivo desse trabalho foi relatar o uso de inibidores da fosfodiesterase nas condições de sepse observando as metodologias empregas, desfechos dos estudos e perspectivas futuras.

\section{Metodologia}

O presente trabalho trata-se de uma revisão integrativa de literatura, de caráter qualitativo (Pereira et al., 2018). Segundo Ercole, Melo \& Alcoforado (2014), essa metodologia permite a demonstração de resultados de pesquisas de forma sistemática, ordenada e abrangente, sendo uma ferramenta que fornece os melhores conhecimentos produzidos para um determinado problema. Para isso, foram incluidos artigos publicados na base de dados MEDLINE/PubMed até outubro de 2020. Os termos 
utilizados na pesquisa foram: "phosphodiesterase AND sepsis" obtendo 372 resultados. Após triagem inicial de tipo de artigo, título e resumo, 20 estudos pré-clínicos foram selecionados para serem descritos.

\section{Resultados e Discussão}

\section{Sepse e choque séptico: definições}

A sepse é uma síndrome clínica que representa a principal causa de morte por infecção em hospitais (Singer et al., 2016). O termo sepse foi definido pela primeira vez pela American College of Chest Physicians (CHEST) e pela Society of Critical Care Medicine (SCCM) em 1992 como uma resposta sistêmica à infecção. Nesse documento, a Síndrome da Resposta Inflamatória Sistêmica (SIRS) foi caracterizada por temperatura corporal acima de $38^{\circ} \mathrm{C}$ ou menos que $36^{\circ} \mathrm{C}$, frequência cardíaca maior que 90 batimentos por minuto, taquipneia ou hiperventilação e alteração na contagem de células brancas (maior que $12.000 / \mathrm{mm}^{3}$ ou menor que $4000 / \mathrm{mm}^{3}$ ). Pacientes que apresentassem dois ou mais critérios SIRS combinados a suspeita de infecção tinham diagnóstico clínico para sepse, sendo que o termo sepse grave foi utilizado para definir disfunção orgânica como resultado da sepse. Já o choque séptico foi definido como hipotensão ou hiperlactatemia persistentes após tentativa de ressuscitação com fluídos (Bone et al., 1992).

A Conferência Internacional de Definições de Sepse de 2001 discutiu a necessidade de atualização do termo sepse, conforme as descobertas sobre a sua fisiopatologia avançavam. A principal crítica da conferência foi em relação ao uso do critério SIRS para diagnóstico (Levy et al., 2001). O método se mostrou altamente sensível para inflamação e pouco específico para sepse, pois os pacientes podiam apresentar os 4 critérios mesmo sem infecção (Cecconi et al., 2018). No documento publicado foi sugerido uma nova ferramenta de classificação da sepse denominada PIRO, levando em consideração as condições de prédisposição do paciente, a natureza e extensão da infecção, a natureza e a magnitude da resposta do hospedeiro e o grau de disfunção orgânica concomitante (Levy et al., 2001).

Somente em 2014, a SCCM e a European Society of Intensive Care Medicine (ESICM) reuniu 19 especialistas para reexaminar as definições vigentes. Sepse foi então definida como uma disfunção orgânica com risco de vida causada por uma resposta desregulada do hospedeiro à infecção, enfatizando a resposta não-homeostática do hospedeiro, a alta mortalidade e necessidade de reconhecimento precoce. Nesse contexto, o termo "sepse grave" se tornou redundante e foi descontinuado. Choque séptico ficou definido como um subconjunto da sepse, em que o sistema circulatório e as anormalidades celulares e metabólicas são profundas o suficiente para aumentar a mortalidade (Singer et al., 2016).

$\mathrm{O}$ termo SIRS também foi descontinuado devido à baixa evidência que os critérios apresentavam. Em um estudo retrospectivo, 12,1\% de 109.663 pacientes internados com infecção e falência de órgãos não atingiram dois critérios SIRS entre 2000 e 2013 na Austrália (Kaukonen et al., 2015). Diante disso, os especialistas recomendaram a utilização da avaliação sequencial de falência de órgãos (SOFA) para melhorar o reconhecimento de pacientes críticos. A pontuação SOFA leva em consideração índice de respiração ( $\mathrm{PaO} 2 / \mathrm{FiO} 2)$, sistema cardiovascular (pressão arterial média), função hepática (nível de bilirrubina), estado de coagulação (contagem de plaquetas), função renal (nível de creatinina ou débito urinário) e estado neurológico (escala de coma de Glasgow) (Vafaei et al., 2019). Uma mudança aguda maior que 2 pontos na pontuação SOFA identifica uma disfunção orgânica. Já o choque séptico permanece como hipotensão que exige vasopressores para manter a pressão arterial média maior ou igual a $65 \mathrm{mmHg}$ e nível de lactato sérico maior que $2 \mathrm{mmol} / \mathrm{L}$ apesar de ressuscitação com volume adequado de fluídos (Singer et al., 2016).

\section{Epidemiologia}

Dados globais da incidência e mortalidade da sepse são difíceis de serem obtidos, os arquivos hospitalares nem sempre refletem a quantidade real de casos. Um estudo que utilizou dados do Global Burden of Diseases (GBD) estimou que 48,9 
milhões de casos de sepse foram registrados em todo o mundo em 2017. Além disso, 11,0 milhões de mortes foram relatadas, representando 19,7\% de todas as mortes globais daquele ano (Rudd et al., 2020). Ainda em 2017, a Organização Mundial as Saúde (OMS) declarou a sepse como uma prioridade na saúde global destacando a necessidade de melhorar a prevenção, diagnóstico e tratamento da síndrome (Kumar, 2020). A sepse é, ainda, um problema de saúde pública, chegando a custar 23,7 bilhões de dólares nos Estados Unidos no ano de 2013 (Mcveigh, 2020).

\section{Fisiopatologia}

A fisiopatologia da sepse é um processo complexo, sendo que a resposta desregulada do hospedeiro frente a uma infecção está relacionada a predisposição genética, estado imunológico, idade e comorbidades do paciente, além do tipo de patógeno, extensão e local da infecção (Coopersmith et al., 2018). O Estudo de Prevalência Estendida de Infecção em Terapia Intensiva (EPIC II) constatou que os locais mais comuns de infecção relacionada a sepse são: pulmão (64\%), abdômen (20\%), sangue (15\%) e trato urinário (14\%) (Vincent et al., 2009).

Inicialmente a resposta imune à infecção em pacientes sépticos é caracterizada por uma produção descontrolada de citocinas pró-inflamatórias (TNF- $\alpha$, IL-1, IL-8, IL-12) e anti-inflamatórias (IL-4, IL-10, TGF). Depois disso, o paciente passa para um estágio imunossuprimido (Kumar, 2020; Coopersmith et al., 2018). O sistema circulatório também sofre mudanças durante a sepse e choque séptico, passando de uma vasoconstrição inicial intensa para vasodilatação generalizada que pode não responder a vasoconstritores naturais, gerando hipotensão e hipoperfusão (Kovalski et al., 2017; Russell, Rush \& Boyd, 2018).

Alterações no endotélio tem um papel importante na etiologia da sepse. O aumento da adesão de leucócitos induz um estado pró-coagulante, que combinados a expressão de fator tecidual e deposição de fibrina podem induzir coagulação intravascular disseminada (DIC) além da formação de microtrombos e lesões isquêmicas (Gotts \& Matthay, 2016). A vasodilatação característica da síndrome, pode ser mediada pelo aumento da síntese de óxido nítrico sintase independente de cálcio pelas células vasculares endoteliais e pela liberação de prostaciclinas em resposta a endotoxina e citocinas inflamatórias, além disso, o músculo liso vascular não responde aos mecanismos de vasoconstrição (Russel, Rush \& Boyd, 2018). O endotélio tem sua permeabilidade aumentada e perde a função de barreira, resultando em edema de tecido. Nos pulmões, o acúmulo de edema diminui perfusão e ventilação, reduzindo a complacência pulmonar, além disso, o fluxo sanguíneo capilar também é bloqueado para os demais órgãos, causando difusão de oxigênio prejudicada e disfunção orgânica letal generalizada (Gotts \& Matthay, 2016).

\section{Tratamento}

Diante da grande heterogeneidade da sepse, não existe um tratamento padrão para todos os tipos de pacientes. O manejo da síndrome consiste em realizar o diagnóstico precocemente, ressuscitação com fluídos, administração rápida de antibióticos, ventilação e proteção pulmonar, manutenção da pressão arterial e estratégia para reduzir infecções hospitalares (Gotts \& Matthay, 2016).

De acordo com as recomendações da Campanha Sobrevivendo à Sepse (SSC) de 2017, antibióticos intravenosos de amplo espectro devem ser iniciados até uma hora após o reconhecimento da sepse. Com o resultado da cultura biológica, o espectro do antibiótico deve ser reduzido de acordo com a sensibilidade do patógeno (Rhodes et al., 2017) A ressuscitação com fluídos intravenosos é realizada com o intuito de aumentar o débito cardíaco e melhorar a perfusão de tecidos. A recomendação atual é a utilização de $30 \mathrm{~mL} / \mathrm{Kg}$ de cristalóide IV dentro das primeiras 3 horas de sepse (Singer et al., 2016). Entretanto, alguns pacientes não respondem a essa prática e existe o risco de acúmulo de fluído, agravando o edema (Coopersmith et al., 2018).

Ao longo dos anos, catecolaminas e outras drogas vasopressoras tem sido empregada a fim de reverter a vasodilatação. A norepinefrina tem demonstrado maior segurança e é utilizada como primeira escolha em casos de hipotensão. A outras 
catecolaminas não são recomendadas pelo risco de taquicardias e arritmias. Além disso, a vasopressina tem sido recomendada para reduzir dose de catecolaminas e atingir meta de pressão arterial. Apesar de serem amplamente utilizados, todos os vasopressores podem causar vasoconstrição excessiva e contribuir para disfunção orgânica (Russell, Rush \& Boyd, 2018). Os estudos clínicos de novas estratégias terapêuticas têm dificuldade em mostrar resultados positivos com relevância clínica (Cecconi et al., 2018). Mais de 100 ensaios clínicos randomizados utilizaram tratamentos com foco na modulação da resposta inflamatória, nenhum deles resultou em uma nova terapia (Marshall, 2014). Portanto, a heterogeneidade da doença é um desafio para o estabelecimento de tratamentos unificados (Cecconi et al., 2018).

\section{Inibidores da fosfodiesterase}

As fosfodiesterases (PDE) são enzimas responsáveis por metabolizar as moléculas monofosfato cíclico de adenosina $(\mathrm{AMPc})$ e monofosfato cíclico de guanosina (GMPc), que são segundos mensageiros que atuam em várias vias de sinalização intracelular, modulando diversos efeitos fisiológicos (Derici et al., 2019). O AMPc é produzido pela adenilato ciclase (AC) e ativa a proteína quinase A (PKA), enquanto o GMPc é produzido pela guanilato ciclase (GC) e ativa principalmente a proteína quinase G (PKG) (Andersson, 2018). As PDE podem inativar AMPc e GMPc modulando a amplitude e duração dos seus sinais.

Existem 11 famílias de PDE, com diferentes afinidades por AMPc e GMPc. As PDE-1, PDE-2, PDE-3, PDE-10 e PDE11 agem em ambos nucleotídeos cíclicos. As PDE-4, PDE-7 e PDE-8 atuam principalmente em AMPc e as PDE-5, PDE-6 e PDE-9 em GMPc. As famílias de PDE podem ser expressas em diferentes tecidos, assim, os fármacos desenvolvidos como inibidores da PDE podem tem vários sítios de ação (Derici et al., 2019).

Os inibidores da fosfodiesterase (iPDE) podem gerar vários efeitos fisiológicos decorrentes do aumento de AMPc e/ou GMPc, entre as propriedades farmacológicas dos iPDE se destacam: supressão de TNF-alfa e outras citocinas antiinflamatórias (Németh et al., 1997; Lu et al., 2020), diminuição da permeabilidade endotelial (Fakioglu et al., 2004), diminuição de resistência vascular e melhora da hemodinâmica (Pammi \& Haque, 2004; Flemming et al., 2014), aumento da contração cardíaca (Sakai et al., 2017) e diminuição de fatores pró-coagulantes (Chang, 2015). Esses efeitos podem contribuir para uma melhora no quadro séptico, sendo assim, os iPDE são agentes terapêuticos a serem considerados.

O uso dos iPDE na sepse já é explorado há mais de três décadas. Em 1988, foi publicado um estudo sobre os efeitos hemodinâmicos da amrinona em cães induzidos a choque por endotoxinas. O fármaco, que é um iPDE-3, foi capaz de aumentar o fornecimento e consumo de oxigênio nos cães, além de diminuir a PaO2 (Vincent et al., 1988). Em um estudo clínico, a enoximona, iPDE-3 utilizado na insuficiência cardíaca, melhorou a hemodinâmica em choque cardiogênico pós-cirurgico. Seis pacientes sépticos não sobreviveram, enquanto 7 tiveram alta hospitalar (Iversen et al., 1992). Em um estudo prospectivo randomizado, 48 pacientes com choque séptico receberam dobutamina ou enoximona além da ressuscitação fluída. A enoximona demonstrou propriedades anti-inflamatórias e melhorou a função hepatoesplâncica após 12 horas de tratamento, sendo benéfica comparada com a dobutamina (Kern et al., 2001).

Outro estudo relatou dois casos de choque séptico pediátrico, em que as crianças apresentaram hemorragia adrenal bilateral e coagulopatia. A enoximona melhorou a função cardiovascular pelo aumento da contralitidade e diminuição da resitência vascular. Foram utilizadas catecolaminas após a administração de enoximona, o que provavelmente evitou efeitos colaterais relacionados a vasodilatação. Ambos os pacientes sobreviveram sem sequelas (Ringe, Varnholt \& Gaedicke, 2003).

A milrinona, outro fármaco iPDE-3, também foi utilizada em um ensaio prospectivo controlado por placebo com 12 pacientes pediátricos com choque séptico (Barton et al., 1996). A droga, quando administrada junto com catecolaminas, foi capaz de melhorar a função cardiovascular (índice cardíaco, índice de volume sistólico e entrega de oxigênio) sem apresentar efeitos adversos. Além disso, em 40 pacientes sépticos com depressão miocárdica (Schmittinger et al., 2009), milrinona foi administrada em combinação com metoprolol. Foi observado um índice cardíaco estável com menor frequência cardíaca e maior 
índice de volume sistólico.

A levosimendana, um iPDE-3 com ação sensibilizador de cálcio, age tanto na contratilidade miocárdica, quanto na proteção hepática e renal, sendo benéfico em pacientes com sepse e choque séptico (Pan et al., 2019). Dados de um estudo prospectivo randomizado controlado com 36 pacientes demonstraram que a levosimendana pode aumentar função cardíaca e índices hemodinâmicos sistêmicos em pacientes com choque séptico (Fang \& Dong, 2014). Sete ensaios clínicos, com 246 pacientes com sepse ou choque séptico no total, foram avaliados em uma meta-análise. A levosimendana revelou estar associada a uma menor mortalidade em comparação com a dobutamina (terapia inotrópica padrão) (Zangrillo et al., 2015).

Além dos fármacos iPDE-3, a pentoxifilina (PTX) é um inibidor não seletivo da PDE que se destaca no tratamento da sepse. A PTX possui ação predominante em AMPc, e é usado principalmente em casos de insuficiência circulatória, como na claudicação intermitente. Em 1992, foi publicado um estudo que comparou os efeitos da administração de pentoxifilina ou ibuprofeno antes da aplicação de endotoxina em humanos. Todos os grupos tiveram alterações hemodinâmicas (aumento de frequência cardíaca e índice cardíaco e diminuição da resistência vascular sistêmica), portanto a resposta hemodinâmica cardiovascular à administração de endotoxina em humanos não melhorou com ibuprofeno ou pentoxifilina (Martich et al., 1992). Entretanto, em 1998, um estudo randomizado controlado por placebo revelou que a administração contínua de PTX por 28 dias reduziu a disfunção cardiopulmonar a partir do quarto dia em pacientes sépticos. Cinquenta e um pacientes participaram do estudo, e a administração de PTX não foi relacionada a efeitos adversos (Staubach et al., 1988).

Recentemente, uma meta análise de 7 estudos randomizados controlados, analisou a eficácia da PTX no tratamento da sepse neonatal. Em uma amostra de 439 neonatos, a PTX mostrou não reduzir significativamente a mortalidade por sepse neonatal, entretanto, interferiu na diminuição do tempo de permanência hospitalar, então a sua utilização pode ser benéfica (Tian et al., 2019). Um outro inibidor não seletivo da PDE, teofilina, também demonstrou ser benéfico ao ser associado a um menor risco de mortalidade relacionada a sepse em pacientes com doença pulmonar obstrutiva crônica (Shih et al., 2017).

Apesar dos resultados promissores, os estudos com os iPDE foram realizados com um baixo tamanho amostral, o que fornece um baixo nível de evidência para que essa classe de medicamentos seja utilizada como tratamento padrão da sepse e choque séptico. Os estudos clínicos com pacientes sépticos possuem várias limitações visto que esses pacientes tem a característica de possuir um alto número de comorbidades e uma alta heterogeneidade, respondendo de forma diferente ao tratamento, o que dificulta a padronização de resultados. Além disso, a sepse é uma síndrome de alta gravidade que exige intervenção imediata (Hollenberg, 2005). Diante disso, estudos pré-clínicos em modelos animais são importantes para o esclarecimento de mecanismos envolvidos na sepse, bem como para o desenvolvimento de novas abordagens terapêuticas (Hollenberg, 2005; Korneev, 2019).

\section{Modelos animais}

A injeção de lipopolissacarídeos (LPS) purificados provenientes de bactérias, como Escherichia coli, via intraperitoneal ou intravenosa é uma maneira de induzir endotoxemia em ratos e camundongos. A infusão da endotoxina está relacionada a diminuição do débito cardíaco e aumento da resistência vascular periférica (Cryer, Garrison \& Harris, 1988). Esse modelo é relacionado à alta mortalidade dos animais dentro das primeiras 12 horas, sendo preconizado para o estudo de eventos agudos na sepse (Piper et al., 1996).

A ligadura e punção cecal (CLP) em roedores é o modelo usado com maior frequência. Ele consiste em um foco infeccioso polimicrobiano intra-abdominal. Após a punção, ocorre translocação bacteriana para o sangue do animal, gerando resposta inflamatória (Rittisch et al., 2009). O tempo de sobrevivência dos animais é geralmente maior em comparação ao modelo LPS, sendo que após 24 horas da indução da sepse a taxa de mortalidade é em média 50\% (Sakai et al., 2017) permitindo um estudo mais prolongado das características hemodinâmicas dos animais (Hollenberg, 2005). 
Um outro modelo denominado "two-hit trauma" tem sido utilizado como forma de mimetizar a prática clínica (Murphy et al., 2005). Nesse modelo, os animais são submetidos a um trauma inicial, geralmente uma queimadura de terceiro grau por contato com água em temperatura próxima a $100^{\circ} \mathrm{C}$. Posteriormente, o segundo trauma é estabelecido por CLP ou injeção de LPS, gerando a uma resposta inflamatória sistêmica exagerada com maior chance de desenvolver falência múltipla de órgãos (Özer, Topal \& Sem, 2020; Paterson et al., 2003).

\section{Estudos pré-clínicos com agentes inibidores da fosfodiesterase na sepse e choque séptico}

Entre as 11 famílias de PDE que se conhece atualmente (Derici et al., 2019), os estudos em modelos animais sépticos têm foco principalmente nos fármacos inibidores não seletivos da PDE (Quadro 1), nos inibidores da PDE-3 (Quadro 2), inibidores da PDE-4 (Quadro 3) e inibidores da PDE-5 (Quadro 4).

Quadro 1. Estudos em modelos sépticos utilizando inibidores não seletivos da fosfodiesterase.

\begin{tabular}{|c|c|c|c|c|}
\hline Artigo & Fármaco & Dose & Modelo & Principais achados \\
\hline Fakioglu et al., 2004 & Aminofilina & $\begin{array}{c}1 \mathrm{mg} / \mathrm{kg}+0,5 \mathrm{mg} / \mathrm{kg} / \mathrm{h} \\
\text { (imediatamente após injeção } \\
\text { de LPS) }\end{array}$ & $\begin{array}{l}\text { Infusão de } \\
\text { LPS em } \\
\text { murinos }\end{array}$ & $\begin{array}{c}\text { Falha na prevenção de } \\
\text { manifestações } \\
\text { respiratórias e } \\
\text { hemodinâmicas. }\end{array}$ \\
\hline Harada et al., 1989 & Aminofilina & $\begin{array}{c}5 \mathrm{mg} / \mathrm{kg}+1,25 \mathrm{mg} / \mathrm{kg} / \mathrm{h} \text { e } 20 \\
\mathrm{mg} / \mathrm{kg}+6 \mathrm{mg} / \mathrm{kg} / \mathrm{h} \\
(1 \mathrm{~h} \text { antes da injeção de } E . \\
\text { coli })\end{array}$ & $\begin{array}{l}\text { Infusão de } \\
\text { Escherichia } \\
\text { coli em } \\
\text { porquinhos-da } \\
\quad \text { índia }\end{array}$ & $\begin{array}{l}\text { Prevenção do acúmulo } \\
\text { tecidual de albumina, } \\
\text { porém com diminuição } \\
\text { da pressão arterial média } \\
\text { (principalmente com a } \\
\text { maior dose). }\end{array}$ \\
\hline Harada et al., 1989 & Pentoxifilina & $\begin{array}{c}20 \mathrm{mg} / \mathrm{kg}+6 \mathrm{mg} / \mathrm{kg} / \mathrm{h} \\
(1 \mathrm{~h} \text { antes da injeção de } E . \\
\text { coli) }\end{array}$ & $\begin{array}{l}\text { Infusão de } \\
\text { Escherichia } \\
\text { coli em } \\
\text { porquinhos-da } \\
\quad \text { índia }\end{array}$ & $\begin{array}{l}\text { Prevenção do acúmulo } \\
\text { tecidual de albumina }\end{array}$ \\
\hline Németh et al., 1997 & Teofilina & $\begin{array}{c}100 \mathrm{mg} / \mathrm{kg} \text { (30 min antes da } \\
\text { injeção de LPS) }\end{array}$ & $\begin{array}{l}\text { Infusão de } \\
\text { LPS em } \\
\text { murinos }\end{array}$ & $\begin{array}{l}\text { Diminuição de TNF- } \alpha \text { e } \\
\text { NO. IL-6 e IL-10 altos } \\
\text { durante toda a resposta }\end{array}$ \\
\hline $\begin{array}{c}\text { Özer, Topal \& Şen, } \\
2020\end{array}$ & Pentoxifilina & $\begin{array}{c}50 \mathrm{mg} / \mathrm{kg} / \text { dia i.p ( } 72 \text { horas } \\
\text { após CLP) }\end{array}$ & $\begin{array}{l}\text { Queimadura + } \\
\text { CLP em } \\
\text { murinos } \\
\text { "Two-hit } \\
\text { trauma" }\end{array}$ & $\begin{array}{l}\text { Diminuição de estresse } \\
\text { oxidativo e lesão } \\
\text { orgânica. Aumento do } \\
\text { nível de SOD no rim e } \\
\text { diminuição de TNF- } \alpha \text {. }\end{array}$ \\
\hline
\end{tabular}

Fonte: Adaptado de Fakioglu et al. (2004), Harada et al. (1989), Németh et al. (1997) e Özer, Topal \& Sen (2020).

A aminofilina e teofilina possuem efeitos broncodilatadores, podendo ser utilizados como anti-asmáticos, na redução da hipertensão pulmonar e fibrose pulmonar (Fakioglu et al., 2004). Enquanto a pentoxifilina tem uma ação um pouco diferente dos outros iPDE-ns, atuando na insuficiência vascular periférica. Acredita-se que os iPDE-ns são capazes de reduzir a permeabilidade endotelial. Esse efeito é atribuído ao aumento de AMPc intracelular e inibição de TNF- $\alpha$. No estudo de Harada et al. (1989), esse efeito é demonstrado pela diminuição do vazamento de albumina em diversos órgãos de cobaias submetidas à um modelo de sepse por infusão de Escherichia coli. Diante disso, concluiu-se que pentoxifilina e aminofilina poderiam ter um 
efeito benéfico em prevenir lesão tecidual em condições sépticas. Em 2004, Fakioglu e colaboradores demonstraram que a aminofilina falhou em prevenir a lesão pulmonar em um modelo de ratos endotoxemicos. Nesse estudo, o fármaco foi administrado imediatamente após a aplicação da endotoxina, sendo que no modelo de Harada et al. (1989), a aminofilina foi administrada antes da indução de endotoxemia, o que pode ter influenciado nos resultados.

A pentoxifilina, por outro lado, em um estudo mais recente mostrou diminuir os scores de dano tecidual por meio da histopatologia do pulmão e rim de ratos submetidos a um modelo de "two-hit trauma" (Özer, Topal \& Sem, 2020). Nos estudos pré-clínicos, a aminofilina demonstra diminuir a pressão arterial média (PAM) dos animais, o que pode ser prejudicial em situações de sepse, em que a hipotensão já é um efeito presente. Já a pentoxifilina demonstra não interferir na PAM dos animais, podendo ser uma vantagem na farmacoterapia da sepse e choque séptico (Harada et al., 1989). A teofilina apresentou um resultado controverso quando administrada meia hora antes da injeção de LPS em ratos, ao aumentar concentração da citocina pró-inflamatória IL-6 e da citocina anti-inflamatória IL-10. Entretanto, demonstrou diminuir a concentração de TNF- $\alpha$ e metabólitos do óxido nítrico, mediadores pró-inflamatórios endógenos derivados de macrófagos que estão diretamente relacionados a lesão orgânica e hiporeatividade vascular a vasoconstritores (Németh et al., 1997).

Quadro 2. Estudos em modelos sépticos utilizando inibidores da fosfodiesterase tipo 3.

\begin{tabular}{|c|c|c|c|c|}
\hline Artigo & Fármaco & Dose & Modelo & Principais achados \\
\hline Németh et al., 1997 & Amrinona & $\begin{array}{l}100 \mathrm{mg} / \mathrm{kg}(30 \mathrm{~min} \\
\text { antes da injeção de } \\
\text { LPS })\end{array}$ & $\begin{array}{l}\text { Infusão de LPS } \\
\text { em murinos }\end{array}$ & $\begin{array}{l}\text { Diminuição de TNF- } \alpha \text { e NO. } \\
\text { IL-6 e IL-10 se elevam na } \\
\text { resposta secundária }\end{array}$ \\
\hline $\begin{array}{l}\text { Yamashita et al., } \\
2018\end{array}$ & Milrinona & $\begin{array}{l}0,5 \mu \mathrm{g} / \mathrm{kg} / \mathrm{min} \text { i.p. } \\
\text { (imediatamente após } \\
\text { CLP) }\end{array}$ & $\begin{array}{l}\text { CLP em } \\
\text { murinos }\end{array}$ & $\begin{array}{c}\text { Aumento da inflamação } \\
\text { cardíaca (TNF- } \alpha \text {, IL-1 e IL-6) }\end{array}$ \\
\hline Schmidt et al., 2000 & Amrinona & $\begin{array}{c}40 \mu \mathrm{g} / \mathrm{kg} / \mathrm{min} \text { i.v. (30 } \\
\text { min antes da injeção de } \\
\text { LPS) }\end{array}$ & $\begin{array}{l}\text { Infusão de LPS } \\
\text { em murinos }\end{array}$ & $\begin{array}{l}\text { Prevenção da hipóxia tecidual } \\
\text { intestinal, porém com } \\
\text { diminuição da pressão arterial } \\
\text { média. }\end{array}$ \\
\hline Schmidt et al., $2000^{\mathrm{b}}$ & Milrinona & $\begin{array}{l}0,5 \mu \mathrm{g} / \mathrm{kg} / \mathrm{min} \text { i.v. (30 } \\
\text { min antes da injeção de } \\
\text { LPS) }\end{array}$ & $\begin{array}{l}\text { Infusão de LPS } \\
\text { em murinos }\end{array}$ & $\begin{array}{c}\text { Prevenção da vasoconstrição } \\
\text { das vilosidades intestinais, } \\
\text { porém com diminuição da } \\
\text { pressão arterial média. }\end{array}$ \\
\hline $\begin{array}{l}\text { Miyakawa et al., } \\
2004\end{array}$ & Olprinona & $\begin{array}{c}15 \text { mg/kg i.p. (1 h antes } \\
\text { da CLP) }\end{array}$ & $\begin{array}{l}\text { CLP em } \\
\text { murinos }\end{array}$ & $\begin{array}{l}\text { Inibição da atividade de } \\
\text { mieloperoxidase e melhora da } \\
\text { fatigabilidade diafragmárica. }\end{array}$ \\
\hline Sakai et al., 2017 & Milrinona & $\begin{array}{l}\text { 0,01 mg/kg i.v. } \\
\text { (imediatamente após } \\
\text { CLP) }\end{array}$ & $\begin{array}{l}\text { CLP em } \\
\text { murinos }\end{array}$ & $\begin{array}{c}\text { Manutenção de efeitos } \\
\text { ionotrópicos (em comparação } \\
\text { com falha da dobutamina). }\end{array}$ \\
\hline
\end{tabular}




\begin{tabular}{|c|c|c|c|c|}
\hline Takeuchi et al., 1998 & Amrinona & $\begin{array}{c}1 \mathrm{mg} / \mathrm{kg}+10 \\
\mu \mathrm{g} / \mathrm{kg} / \mathrm{min} \text { i.v. } \\
\text { (imediatamente após a } \\
\text { injeção de LPS) }\end{array}$ & $\begin{array}{l}\text { Infusão de LPS } \\
\text { em coelhos }\end{array}$ & $\begin{array}{l}\text { Diminuição de TNF- } \alpha \text {, IL-1 e } \\
\text { atividade de iNOs. }\end{array}$ \\
\hline Chang, 2015 & Cilostazol & $\begin{array}{l}10 \mathrm{mg} / \mathrm{kg}(2 \mathrm{~h} \text { antes e } \\
12 \mathrm{~h} \text { depois da CLP })\end{array}$ & $\begin{array}{l}\text { CLP em } \\
\text { murinos }\end{array}$ & $\begin{array}{l}\text { Diminuição da liberação de } \\
\text { HMGB1. Aumento da } \\
\text { sobrevivência. }\end{array}$ \\
\hline Haskó et al., 1988 & Amrinona & $\begin{array}{l}10 \mathrm{~mL} / \mathrm{kg}(30 \mathrm{~min} \\
\text { antes da injeção } \\
\text { de LPS })\end{array}$ & $\begin{array}{l}\text { Infusão de LPS } \\
\text { em murinos }\end{array}$ & $\begin{array}{l}\text { Inibição de IL-12, IFN- } \gamma, \\
\text { TNF } \alpha .\end{array}$ \\
\hline $\begin{array}{c}\text { Özer, Topal \& Şen, } \\
2020\end{array}$ & Milrinona & $\begin{array}{l}1 \mathrm{mg} / \mathrm{kg} \text { i.p. ( } 72 \\
\text { horas após CLP) }\end{array}$ & $\begin{array}{l}\text { Queimadura + } \\
\text { CLP em } \\
\text { murinos } \\
\text { "Twohit } \\
\text { trauma" }\end{array}$ & $\begin{array}{l}\text { Diminuição de estresse } \\
\text { oxidativo e lesão orgânica. } \\
\text { Aumento de glutationa } \\
\text { peroxidase (GPx) renal, e } \\
\text { SOD pulmonar. Diminuição } \\
\text { de TNF- } \alpha \text {. }\end{array}$ \\
\hline
\end{tabular}

Fonte: Adaptado de Németh et al. (1997), Yamashita et al. (2018), Schmidt et al. (2000)a, Schmidt et al. (2000) ${ }^{\text {b }}$, Miyakawa et al. (2004), Sakai et al. (2017), Takeuchi et al. (1998), Chang (2015), Haskó et al. (1998) e Özer, Topal \& Sen (2020).

Os fármacos iPDE-3 possuem propriedades ionotrópicas, sendo utilizados na insuficiência cardíaca. A dobutamina, um ionotrópico agonista $\beta 1$ adrenérgico, é a primeira linha de tratamento no choque séptico (Rhodes et al., 2017). Diante das propriedades anti-inflamatórias e cardioprotetoras, os iPDE-3 podem oferecer benefícios em situações de sepse e choque séptico, sendo testados em vários modelos e comparados com a dobutamina (Sakai et al., 2017; Yamashita et al., 2018). A milrinona foi administrada logo após a cirurgia de CLP em ratos, ao contrário do esperado, foi observando o aumento das citocinas próinflamatórias TNF- $\alpha$, IL-1 e IL-6 no tecido cardíaco. Apesar disso, ouve a diminuição de marcadores de lesão cardíaca, como troponina-I (Yamashita et al., 2018). Em outro estudo, a milrinona demonstrou exercer efeitos ionotrópicos mesmo em situações de sepse, enquanto a dobutamina se mostrou inferior em aumentar o débito cardíaco nas mesmas condições. A atividade inflamatória do fármaco não foi relatada, porém, foi observado que a milrinona tem a capacidade de inibir tanto a PDE-3 quanto a PDE-4, e as propriedades anti-inflamatórias dos iPDE-4 são documentadas in vitro e in vivo (Sakai et al., 2017; Montana \& Dyke, 2002). Apesar dos efeitos benéficos, a milrinona possui efeitos vasodilatadores, que foram observados no estudo de Schmidt et al. (2000)b por meio da diminuição da MAP, podendo ser uma desvantagem do seu uso.

A amrinona comprovou reduzir citocinas pró-inflamatórias (TNF- $\alpha$, IL-1, IL-6, IL-12, e IFN- $\gamma$ ) em roedores endotoxemicos (Németh et al., 1997; Takeuchi et al., 1998; Haskó et al., 1988). Esses efeitos foram associados a uma melhora na perfusão tecidual, prevenindo isquemia e lesão tecidual. Por outro lado, a amrinona também contribuiu para a hipotensão observados pela diminuição da MAP durante a endotoxemia. Dessa forma o uso desses iPDE-3 na sepse deve ser considerado em associação com agentes vasoconstritores, para evitar a queda da pressão arterial (Schimidt et al., 2000a). O fármaco olprinona, que também age como ionotrópico e vasodilatador, foi administrado previamente a CLP em ratos, para a investigação de seu potencial em prevenir insuficiência respiratória e fatigabilidade diafragmática. A olprinona demonstrou ter um efeito benéfico agindo provavelmente pela inibição da ativação de neutrófilos e inibição de radicais livres de oxigênio (Miyakawa et al., 2004). Já o fármaco cilostazol possui ação anticoagulante e anti-inflamatória. O estudo de Chang (2015) observou que esse iPDE-3 reduz a liberação da proteína HMGB1, que atua na patogênese da coagulação vascular disseminada, além disso, aumentou a sobrevivência de ratos submetidos a CLP, mostrando ser promissor no tratamento da sepse. 
Quadro 3. Estudos em modelos sépticos utilizando inibidores da fosfodiesterase tipo 4.

\begin{tabular}{|c|c|c|c|c|}
\hline Artigo & Fármaco & Dose & Modelo & Principais achados \\
\hline Haskó et al., 1988 & Rolipram & $\begin{array}{l}10 \mathrm{~mL} / \mathrm{kg} \text { (30 min antes } \\
\text { da injeção de LPS) }\end{array}$ & $\begin{array}{l}\text { Infusão de LPS } \\
\text { em murinos }\end{array}$ & $\begin{array}{c}\text { Inibição de IL-12, IFN- } \gamma, \text { TNF- } \alpha \\
\text { e NO. }\end{array}$ \\
\hline $\begin{array}{l}\text { Holthoff et al., } \\
\quad 2013\end{array}$ & Rolipram & $\begin{array}{l}1 \mathrm{mg} / \mathrm{kg} \text { e } 3 \mathrm{mg} / \mathrm{kg} \text { i.p. } \\
(5,5 \text { horas após CLP) }\end{array}$ & $\begin{array}{l}\text { CLP em } \\
\text { murinos }\end{array}$ & $\begin{array}{l}\text { Diminuição da permeabilidade } \\
\text { vascular, aumento do fluxo } \\
\text { sanguíneo renal, diminuição de } \\
\text { ureia e creatinina. }\end{array}$ \\
\hline $\begin{array}{l}\text { Flemming et al., } \\
\qquad 2014\end{array}$ & Rolipram & $\begin{array}{c}1 \mathrm{mg} / \mathrm{kg} / \mathrm{h} \text { e } 3 \mathrm{mg} / \mathrm{kg} / \mathrm{h} \\
\text { i.v. (12 horas após } \\
\text { CASP) }\end{array}$ & $\begin{array}{l}\text { CASP em } \\
\text { murinos }\end{array}$ & $\begin{array}{c}\text { Estabilização da barreira } \\
\text { microvascular e melhor fluxo } \\
\text { microcirculatório. Diminuição de } \\
\text { IL-1, IL-12 e TNF- } \alpha \text { (a dose baixa } \\
\text { foi eficaz, enquanto a dose de } \\
3 \mathrm{mg} / \mathrm{kg} / \mathrm{h} \text { mostrou efeitos } \\
\text { adversos). }\end{array}$ \\
\hline Sims et al., 2017 & Rolipram & $\begin{array}{l}0,1 \mathrm{mg} / \mathrm{kg} \text { i.p. ( } 6 \text { horas } \\
\text { após CLP) }\end{array}$ & $\begin{array}{c}\text { CLP em } \\
\text { murinos }(18 \\
\text { dias de idade })\end{array}$ & $\begin{array}{l}\text { Restauração da função cardíaca e } \\
\text { microcirculação renal. Redução da } \\
\text { permeabilidade vascular. } \\
\text { Diminuição da mortalidade. }\end{array}$ \\
\hline Feng et al., 2017 & Roflumilaste & $\begin{array}{c}1 \mathrm{mg} / \mathrm{kg}, 5 \mathrm{mg} / \mathrm{kg} \text { ou } 10 \\
\mathrm{mg} / \mathrm{kg} \text { i.p. (1 horas } \\
\text { antes, } 1 \text { hora depois ou } \\
\text { imediatamente após } \\
\text { injeção de LPS). }\end{array}$ & $\begin{array}{l}\text { Infusão de } \\
\text { Escherichia } \\
\text { coli, infusão de } \\
\text { LPS e CLP em } \\
\text { murinos }\end{array}$ & 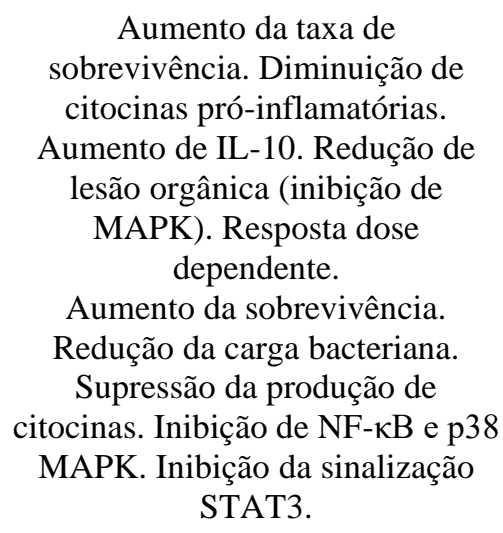 \\
\hline Chang, 2015 & Roflimilaste & $\begin{array}{c}1 \mathrm{mg} / \mathrm{kg} \text { (diariamente, } 7 \\
\text { dias após a injeção de } \\
\text { LPS) }\end{array}$ & $\begin{array}{l}\text { Infusão de LPS } \\
\text { em murinos }\end{array}$ & $\begin{array}{c}\text { Aumento da sobrevivência. } \\
\text { Diminuição de TNF- } \alpha \text {, IL-6, JAK } \\
\text { e STAT3. Menor número de } \\
\text { neutrófilos, monócitos e linfócitos } \\
\text { no líquido pulmonar. }\end{array}$ \\
\hline Xu et al., 2020 & Roflumilaste & $\begin{array}{l}1 \mathrm{mg} / \mathrm{kg} \text { ou } 3 \mathrm{mg} / \mathrm{kg} \\
\text { (diariamente, } 7 \text { dias } \\
\text { antes da CLP) }\end{array}$ & $\begin{array}{l}\text { CLP em } \\
\text { murinos }\end{array}$ & $\begin{array}{c}\text { Diminuição da resposta } \\
\text { inflamatória (IL-1, TNF- } \alpha \text {, IL-6, } \\
\text { NF-אB). Redução de alterações } \\
\text { histopatológicas e marcadores de } \\
\text { lesão renal de maneira dose } \\
\text { dependente. }\end{array}$ \\
\hline
\end{tabular}

Fonte: Adaptado de Haskó et al. (1988), Holthoff et al. (2013), Flemming et al. (2014), Sims et al. (2017), Lu et al. (2020), Feng et al. (2017), Chang (2015) e Xu et al. (2020).

A PDE-4 é amplamente expressa nas células endoteliais, a inibição dessa enzima pode causar efeitos desejados no tratamento da sepse, como a regulação do tônus vascular e diminuição da permeabilidade endotelial (Holthoff et al., 2013). Dessa forma, somado ao potencial anti-inflamatório, os iPDE-4 têm ganhado destaque nos estudos que utilizam modelos de sepse e choque séptico. O fármaco rolipram demonstrou restaurar a perfusão capilar peritubular e melhorar o fluxo sanguíneo renal 
(Holthoff et al., 2013), prevenir edema e lesão pulmonar (Flemming et al., 2014), prevenir síndrome cardiorrenal (Sims et al., 2017) e aumentar a sobrevivência (Lu et al., 2020) de roedores submetidos a diferentes modelos de sepse. Os mecanismos envolvidos são provavelmente: a diminuição da permeabilidade endotelial, a diminuição de citocinas pró-inflamatórias (IL-1, IL-12, NF-кB e TNF- $\alpha$ ), o aumento da citocina anti-inflamatória IL-10, a inibição da proteína ativada por mitogênio quinase (MAPK), que regula a expressão de citocinas em vários níveis e a regulação positiva da proteína de especificidade dupla fosfatase 1 (DUSP1), que inativa vias inflamatórias mediadas por MAPK.

O roflumilaste, que é utilizado no tratamento de doença pulmonar obstrutiva crônica, demonstrou diminuir lesão hepática (Feng et al., 2017), aumentar o tempo de sobrevivência (Chang, 2015; Feng et al., 2017), melhorar a morfologia do tecido pulmonar (Chang, 2015), aliviar lesão renal e danos histopatológicos induzidos por sepse (Xu et al., 2020) em ratos submetidos a CLP ou infusão de LPS. As vias de regulação envolvidas foram: a inibição de IL-6, TNF- $\alpha$ e NF-Kb, fosforilação da MAPK, inibição do fator de transcrição ativados por tirosina quinase 3 (STAT3), uma via intimamente relacionada a inflamação, a qual é ativada na sepse (Chang, 2015). Devido a quantidade de resultados benéficos e mínimos efeitos colaterais, os iPDE-4 têm demonstrado serem agentes promissores no tratamento da sepse.

Quadro 4. Estudos em modelos sépticos utilizando inibidores da fosfodiesterase tipo 5.

\begin{tabular}{|c|c|c|c|c|}
\hline Artigo & Fármaco & Dose & Modelo & Principais achados \\
\hline Cadirci et al., 2011 & Sildenafil & $\begin{array}{c}10 \mathrm{mg} / \mathrm{kg} \text { ou } 20 \mathrm{mg} / \mathrm{kg} \\
\text { (imediatamente após } \\
\text { CLP) }\end{array}$ & $\begin{array}{l}\text { CLP em } \\
\text { murinos }\end{array}$ & $\begin{array}{c}\text { Diminuição de marcadores } \\
\text { inflamatórios (TNF- } \alpha, \text { MPO) e de } \\
\text { oxidação (LPO, SOD). Proteção } \\
\text { pulmonar e renal. }\end{array}$ \\
\hline Kovalski et al., 2017 & Sildenafil & $\begin{array}{c}10 \mathrm{mg} / \mathrm{kg} \text { (8 horas após } \\
\text { CLP) }\end{array}$ & $\begin{array}{l}\text { CLP em } \\
\text { murinos }\end{array}$ & $\begin{array}{c}\text { Aumento de fluxo sanguíneo renal, } \\
\text { redução dos níveis de creatinina, } \\
\text { lactato e MPO pulmonar. } \\
\text { Diminuição de neutrófilos no } \\
\text { pulmão. }\end{array}$ \\
\hline Benli et al., 2017 & Tadalafil & $\begin{array}{c}5 \mathrm{mg} / \mathrm{kg} \text { ou } 10 \mathrm{mg} / \mathrm{kg} \\
\text { (imediatamente após } \\
\text { CLP) }\end{array}$ & $\begin{array}{l}\text { CLP em } \\
\text { murinos }\end{array}$ & $\begin{array}{l}\text { Diminuição de creatinina. } \\
\text { Diminuição de inflamação no tecido } \\
\text { renal. }\end{array}$ \\
\hline $\begin{array}{c}\text { Özer, Topal \& Şen, } \\
2020\end{array}$ & Sildenafil & $\begin{array}{c}10 \text { mg/kg i.p. (72 horas } \\
\text { após CLP) }\end{array}$ & $\begin{array}{l}\text { Queimadura } \\
\text { + CLP em } \\
\text { murinos } \\
\text { "Two-hit } \\
\text { trauma" }\end{array}$ & $\begin{array}{l}\text { Diminuição de estresse oxidativo e } \\
\text { lesão orgânica. Aumento de } \\
\text { glutationa peroxidase (GPx). Menor } \\
\text { valor de malondialdeído (MDA). }\end{array}$ \\
\hline
\end{tabular}

Fonte: Adaptado de Cadirci et al. (2011), Kovalski et al. (2017), Benli et al. (2017) e Özer, Topal \& Sen (2020).

Os iPDE-5 agem principalmente elevando o GMPc. Sendo assim, o SIL é capaz de aumentar os níveis de óxido nítrico circulantes causando vasodilatação. Como a PDE-5 é abundante no músculo vascular liso, esse efeito é observado nos corpos cavernosos, sendo assim, o SIL é amplamente utilizado no tratamento da disfunção erétil (Cadirci et al., 2011). Kovalski et al. (2017) sugerem que o aumento nos níveis de GMPc contribui para a manutenção de fluxo sanguíneo adequado e evita a lesão tecidual decorrente da vasoconstrição que ocorre no início do quadro séptico. No referido estudo, o SIL demonstrou aumentar fluxo sanguíneo renal, reduzir níveis de creatinina e lactato, reduzir sequestro de neutrófilos dentro do pulmão e diminuir mieloperoxidase (MPO) pulmonar em um modelo de ratos submetidos a CLP.

Resultados semelhantes já haviam sido descritos por Cacirdi et al. (2011), em que a administração de SIL imediatamente após a CLP promoveu a diminuição de TNF- $\alpha$, peroxidase lipídica (LPO), MPO, superóxido dismutase (SOD), indicando ser um agente protetor de danos pulmonares e renais em quadros sépticos. Recentemente, o SIL demonstrou um efeito protetor a 
lesão tecidual um modelo "two-hit trauma" (10). O fármaco tadalafil também apresentou bons resultados. Em um modelo de ratos submetidos a CLP, a administração de tadalafil diminuiu a citocina pró-inflamatória IL-6, além da redução da inflamação do tecido renal, observada por meio da histopatologia e diminuição da creatinina e cistatina C. Podendo prevenir danos renais em modelos sépticos (Benli et al., 2017).

\section{Considerações Finais}

Os resultados aqui apresentados permitem concluir que os fármacos inibidores da enzima fosfodiesterase possuem efeitos farmacológicos que podem ser benéficos no tratamento da sepse. Entretanto, existe a necessidade de padronizar o método de administração de medicamentos e os modelos animais utilizados, a fim de obter resultados consistentes que possam ser utilizados em pesquisas clínicas. A sepse é uma síndrome heterogênea, sendo difícil obter um fármaco eficaz para o tratamento de todos os pacientes e suas diferentes condições. Não se sabe se os inibidores da fosfodiesterase oferecem vantagens a todos os tipos de pacientes. Portanto, um sistema de subclassificação da sepse poderia evidenciar as necessidades de cada quadro séptico, permitindo um tratamento específico. Entre as famílias estudadas, existem fármacos não abordados que podem ser alvo para trabalhos futuros. Além disso, ainda existem muitas famílias de inibidores da fosfodiesterase não exploradas no tratamento farmacológico da sepse que podem ser agentes promissores para futuras pesquisas.

\section{Referências}

Andersson, K. E. (2018). PDE5 inhibitors-pharmacology and clinical applications 20 years after sildenafil discovery. Br J Pharmacol, $175(13), 2554-2565$.

Barton, P., Garcia, J., Kouatli, A., Kitchen, L., Zorka, A., Lindsay, C., et al. (1996) Hemodynamic effects of iv milrinone lactate in pediatric patients with septic shock: A prospective, double-blinded, randomized, placebo-controlled, interventional study. Chest, 109(5), 1302-1312.

Benli, E., Ayyildiz, S. N., Cirrik, S., Koktürk, S., Cirakoglu, A., Noyan, T., et al. (2017). The effect of tadalafil therapy on kidney damage caused by sepsis in a polymicrobial septic model induced in rats: a biochemical and histopathological study. Int Braz J Urol, 43(2), 345-355.

Bone, R. C., Balk, R. A., Cerra, F. B., Dellinger, R. P., Fein, A. M., Knaus, W. A., et al. (1992). Definitions for sepsis and organ failure and guidelines for the use of innovative therapies in sepsis. Chest, 101(6), 1644-1655.

Cadirci, E., Halici, Z., Odabasoglu, F., Albayrak, A., Karakus, E., Unal, D., et al. (2011). Sildenafil treatment attenuates lung and kidney injury due to overproduction of oxidant activity in a rat model of sepsis: a biochemical and histopathological study. Clin Exp Immunol, 166(3), 374-384.

Cecconi, M., Evans, L., Lvy, M. \& Rhodes, A. (2018) Sepsis and septic shock. Lancet, 392(10141), 75-87.

Chang, C. K. (2015). Cilostazol inhibits HMGB1 release in LPS-activated RAW 264.7 cells and increases the survival of septic mice. Thromb Res, 136(1), 456464.

Coopersmith, C. M., Baker, D., Deutschman, C. S., Ferrer, R., Lat, I., Machado, F. R., et al. (2018). Surviving sepsis campaign: research priorities for sepsis and septic shock. Intensive Care Med, 44(9), 1400-1426.

Cryer, H. M., Garrison, R. N. \& Harris, P. D. (1988). Role of muscle microvasculature during hyperdynamic and hypodynamic phases of endotoxin shock in decerebrate rats. J Trauma, 28(3), 312-318.

Derici, M. K., Sadi, G., Cenik, B., Güray, T. \& Demirel-Yilmaz, E. (2019). Differential expressions and functions of phosphodiesterase enzymes in different regions of the rat heart. Eur J Pharmacol, 844(5),118-129.

Ercole, F. F., Melo, L. S. D., \& Alcoforado, C. L. G. C. (2014). Revisão integrativa versus revisão sistemática. REME, 18(1), 9-12.

Fakioglu, H., Gelvez, J., Torbati, D., Glover, M. L., Olarte, J. L., Camacho, M. T., et al. (2004). Aminophylline therapy during endotoxemia in anesthetized spontaneously breathing rats. Pharmacol Res, 49(1), 45-50.

Fang, M. \& Dong, S. (2014). Effects of levosimendan on hemodynamics and cardiac function in patients with septic shock. Ann Transl Med, 26(10), 692-696.

Feng, H., Chen, J., Wang, H., Cheng, Y., Zou, Z., Zhong, Q., et al. (2017). Roflumilast reverses polymicrobial sepsis-induced liver damage by inhibiting inflammation in mice. Lab Invest, 97(9), 1008-1019.

Flemming, S., Schlegel, N., Wunder, C., Meir, M., Baar, W., Wollborn, J., et al. (2014). Phosphodiesterase 4 inhibition dose dependently stabilizes microvascular barrier functions and microcirculation in a rodent model of polymicrobial sepsis. Shock, 41(6), 537-545.

Gotts, J. E. \& Matthay, M. A. Sepsis: pathophysiology and clinical management. Bmj, 353,1585. 
Harada, H., Ishizaka, A., Yonemaru, M., Mallick, A. A., Hatherill, J. R., Zheng, H., et al. (1989). The effects of aminophylline and pentoxifylline on multiple organ damage after Escherichia coli sepsis. Am J Respir Crit Care Med, 140(4), 974-980.

Haskó, G., Szabó, C., Németh, Z. H., Salzman, A. L. \& Vizi, E. S. (1988). Suppression of IL-12 production by phosphodiesterase inhibition in murine endotoxemia is IL-10 independent. Eur J Immunol, 28(2), 468-472.

Hollenberg, S. M. (2005). Mouse models of resuscitated shock. Shock, 24, 58-63.

Holthoff, J. H., Wang, Z., Patil, N. K., Gokden, N. \& Mayeux, P. R. (2013). Rolipram improves renal perfusion and function during sepsis in the mouse. $J$ Pharmacol Exp Ther, 347(2), 357-364.

Iversen, S., Mayer, E., Hake, U., Schmiedt, W., Jakob, H. \& Oelert, H. (1992). Efficacy of phosphodiesterase inhibitor enoximone in management of postcardiotomy cardiogenic shock. Scand J Thorac Cardiovasc Surg, 26(2), 143-149.

Kaukonen, K. M., Bailey, M., Pilcher, D., Cooper, D. J. \& Bellomo, R. (2015). Systemic inflammatory response syndrome criteria in defining severe sepsis. $N$ Engl J Med, 372(17), 1629-1638.

Kern, H., Schröder, T., Kaulfuss, M., Martin, M., Kox, W. J. \& Spies, C. D. (2001). Enoximone in contrast to dobutamine improves hepatosplanchnic function in fluid-optimized septic shock patients. Critical Care Med, 29(8), 1519-1525.

Korneev, K. V. (2019). Mouse Models of Sepsis and Septic Shock. Mol Biol, 53(5), 704-717.

Kovalski, V., Prestes, A. P., Oliveira, J. G., Alves, G. F., Colarites, D. F., Mattos, J. E, et al. (2017). Protective role of cGMP in early sepsis. Eur J Pharmacol, $807,174-181$

Kumar, V. (2020). Sepsis roadmap: What we know, what we learned, and where we are going. Clin Immunol, 210, 108264

Levy, M. M., Fink, M. P., Marshall, J. C., Abraham, E., Angus, D., Cook, D., et al. (2003). $2001 \mathrm{sccm} / \mathrm{esicm} / \mathrm{accp} /$ ats/sis international sepsis definitions conference. Intensive Care Med, 29(4), 530-538.

Lu, X., Wang, J., Chen, X., Jiang, Y. \& Pan, Z. K. (2020). Rolipram Protects Mice from Gram-negative Bacterium Escherichia coli-induced Inflammation and Septic Shock. Sci Rep, 10(1), 1-8.

Marshall, J. C. (2014). Why have clinical trials in sepsis failed? Trends Mol Medo, 20(4), 195-203.

Martich, G. D., Parker, M. M., Cunnion, R. E. \& Suffredini, A. F. (1992). Effects of ibuprofen and pentoxifylline on the cardiovascular response of normal humans to endotoxin. J Appl Physiol, 73(3), 925-931.

Mcveigh, S. E. (2020). Sepsis Management in the Emergency Department. Nurs Clin North Am, 55(1), 71-79.

Miyakawa, H., Oishi, K., Hagiwara, S., Kira, S., Kitano, T., Iwasaka, H., et al. (2004). Olprinone improves diaphragmatic contractility and fatigability during abdominal sepsis in a rat model. Acta Anaesthesiol Scand, 48(5), 637-641.

Montana, J. G. \& Dyke, H. J. (2002). Update on the therapeutic potential of PDE4 inhibitors. Expert Opin Investig Drugs, 11(1), 1-13.

Murphy, T. J., Paterson, H. M., Kriynovich, S., Zang, Y., Kurt-Jones, E. A., Mannick, J. A. \& Lederes, J. A. (2005). Linking the "two-hit” response following injury to enhanced TLR4 reactivity. J Leukoc Biol, 77(1), 16-23.

Németh, Z. H., Hasko, G., Szabó, C., Vizi \& E. S. (1997). Amrinone and theophylline differentially regulate cytokine and nitric oxide production in endotoxemic mice. Shock, 7(5), 371-375.

Özer, Ö., Topal, U. \& Sem, M. (2020). The effects of specific and non-specific phosphodiesterase inhibitors and N-acetylcysteine on oxidative stress and remote organ injury in two-hit trauma model. Ulus Travma Acil Cerrahi Derg, 36(4), 517-525.

Pammi, M. \& Haque, N. (2004). Pentoxifylline for treatment of sepsis and necrotizing enterocolitis in neonates. Cochrane Database Syst Rev, 1(3), 1-44.

Pan, J., Yang, Y. M., Zhu, J. Y. \& Lu, Y. Q. (2019). Multiorgan Drug Action of Levosimendan in Critical Illnesses. BioMed Res Int, 2019(1), 1-8.

Paterson, H. M., Murphy, T. J., Purcell, E. J., Shelley, O., Kriynovich, S. J., Lien, E., et al. (2003). Injury primes the innate immune system for enhanced Tolllike receptor reactivity. J Immunol, 171(3), 1473-1483.

Pereira, A. S., Shitsuka, D. M., Parreira, F. J., \& Shitsuka, R. (2018). Metodologia da pesquisa científica. Ed (pp. 3-9). UAB/NTE/UFSM. https://repositorio. ufsm. br/bitstream/handle/1/15824/Lic_Computacao_Metodologia-Pesquisa-Cientifica. pdf.

Piper, R. D., Cook, D. J., Bone, R. C. \& Sibbald, W. J. (1996). Introducing critical appraisal to studies of animal models investigating novel therapies in sepsis. Crit Care Med, 24(12), 2059-2070.

Rhodes, A., Evans, L. E., Alhazzani, W., Levy, M. M., Antonelli, M., Ferrer, R., et al. Surviving sepsis campaign: international guidelines for management of sepsis and septic shock: 2016. Intensive Care Med, 43(3), 304-377.

Ringe, H. I. G., Varnholt, V. \& Gaedicke, G. Cardiac rescue with enoximone in volume and catecholamine refractory septic shock. Pediatr Crit Care Med, 4(4), 471-475.

Rittisch, D., Huber-Lang, M. S., Flierl, M. A. \& Ward, P. A. Immunodesign of experimental sepsis by cecal ligation and puncture. Nat Protoc, 4(1), 31-36.

Rudd, K. E., Johnson, S. C., Agesa, K. M., Shackelford, K. A., Tsoi, D., Kievlan, D. R., et al. (2020). Global, regional, and national sepsis incidence and mortality, 1990-2017: analysis for the Global Burden of Disease Study. Lancet, 395(10219), 200-211. 
Russell, J. A., Rush, B. \& Boyd, J. (2018). Pathophysiology of septic shock. Crit Care Clin, 34(1), 43-61.

Sakai, M., Suzuki, T., Tomita, K., Yamashita, S., Palikhe, S., Hattori, K., et al. Diminished responsiveness to dobutamine as an inotrope in mice with cecal ligation and puncture-induced sepsis: attribution to phosphodiesterase 4 upregulation. Am J Physiol Heart Circ Physiol, 312(6),1224-1237.

${ }^{a}$ Schimidt, W., Tinelli, M., Walther, A., Gebhard, M. M., Martin, E. \& Schimidt, H. (2000). Influence of amrinone on tissue oxygenation of jejunal mucosa during endotoxemia. J Surg Res, 93(1), 9-15.

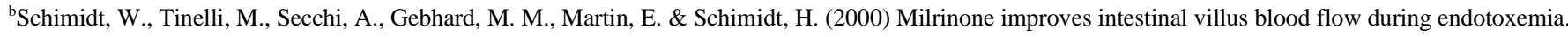
Can J Anaesth, 47(7), 673.

Schmittinger, C. A., Dünser, M. W., Haller, M., Ulmer, H., Luckner, G., Torgersen, C., et al. (2009). Combined milrinone and enteral metoprolol therapy in patients with septic myocardial depression. Crit Care, 12(4), 99.

Shih, Y. N., Chen, Y. T., Chu, H., Shih, C. J., Ou, S. M., Hsu, Y. T., et al. (2017). Association of pre-hospital theophylline use and mortality in chronic obstructive pulmonary disease patients with sepsis. Respir Med, 125, 33-38.

Sims, C. R., Singh, S. P., Mu, S., Gokden, N., Zakaria, D., Nguyen, T. C., et al. (2017). Rolipram improves outcome in a rat model of infant sepsis-induced cardiorenal syndrome. Front Pharmacol, 8, 237.

Singer, M., Deutschman, C. S., Seymour, C. W., Shankar-Hari, M., Annane, D., Bauer, M., et al. (2016). The third international consensus definitions for sepsis and septic shock (Sepsis-3). Jama. 315(8), 801-810.

Staubach, K. H., Schröder, J., Stüber, F., Gehrke, K., Traumann, E. \& Zabel, P. (1988). Effect of pentoxifylline in severe sepsis: results of a randomized, doubleblind, placebo-controlled study. Arch Surg, 133(1), 94-100.

Takeuchi, K., Pedro, J., Ibrahim, A. E., Cao-Danh, H., Friehs, I., Glynn, P., et al. (1998). Vesnarinone and amrinone reduce the systemic inflammatory response syndrome. J Thorac Cardiovasc Surg, 117(2), 375-382.

Tian, J., Shen, P., Pan, K. \& Zhou, Q. (2019). Efficacy of pentoxifylline treatment for neonatal sepsis: a meta-analysis of randomized controlled studies. Riv Ital Pediatr, 45(1), 108 .

Vafaei, A., Heydari, K., Hashemi-Nazari, S. S., Izadi, N., \& Zadeh, H. H. (2019). PIRO, SOFA and MEDS scores in predicting one-month mortality of sepsis patients; a diagnostic accuracy study. Arch Acad Emerg Med, 7(1), e59.

Vincent, J. L., Domb, M., Van der Linden, P., Motte, S., Boelpaepe, C., Contempré, B., et al. (1988). Amrinone administration in endotoxin shock. Circ Shock, 25(2), 75-83.

Vincent, J. L., Rello, J., Marshall, J., Silva, E., Anzueto, A., Martin, C. D., et al. (2009). International study of the prevalence and outcomes of infection in intensive care units. Jama, 302(21), 2323-2329.

Xu, X., Liao, L., Hu, B., Jiang, H. \& Tan, M. (2020). Roflumilast, a Phosphodiesterases-4 (PDE4) Inhibitor, Alleviates Sepsis-induced Acute Kidney Injury. Med Sci Monit, 26, e921319-1.

Yamashita, S., Suzuki, T., Iguchi, K., Sakamoto, T., Tomita, K., Yokoo, H., et al. (2018). Cardioprotective and functional effects of levosimendan and milrinone in mice with cecal ligation and puncture-induced sepsis. Naunyn Schmiedebergs Arch Pharmacol, 391(9), 1021-1032.

Zangrillo, A., Putzu, A., Monaco, F., Oriani, A., Frau, G., De Luca, M., et al. (2015). Levosimendan reduces mortality in patients with severe sepsis and septic shock: a meta-analysis of randomized trials. J Critic Care, 30(5), 908-913. 